\title{
ON THE RATIONAL TRIANGULATION OF A CIRCLE
}

\author{
FRED SUPNICK AND JEANETTE F. KESTON
}

A triangle is called rational if its sides and area are rational. A set of rational triangles is referred to as a rational triangulation of a circle $K$ if (a) each triangle is inscribed in $K$, (b) no two of the triangles have interior points in common, and (c) the sum of the areas of the triangles is equal to the area of $K$.

THEOREM. A circle can be rationally triangulated if and only if its radius is rational.

Proof. If $a, b, c$ are the lengths of the sides of a triangle inscribed in a circle of diameter $k$, then the area of the triangle is

$$
a b c / 2 k \text {. }
$$

Thus, it is impossible to inscribe a rational triangle in a circle having irrational radius.

The case where $K$ has a rational radius is now considered. Let $K$ be given by

$$
r=k \cdot \cos \theta \quad \text { ( } k \text { rational). }
$$

Let $R$ be the set of all numbers $\theta(-\pi / 2<\theta \leqq \pi / 2)$ such that both $\cos \theta$ and $\sin \theta$ are rational, and $S$ the set of all points $(r, \theta)$ on (2) such that $\theta \in R$. The following properties of $S$ will be established:

(i) a point of $S$ can be selected on any subarc of (2);

(ii) any three points of $S$ are the vertices of a rational triangle;

(iii) on any minor subarc $\overparen{A C}$ of (2), a point $B \in S$ can be selected such that the area of $\triangle A B C$ exceeds one-fourth that of the segment $A C A$ (i.e. the segment of (2) bounded by the arc $\overparen{A C}$ and the chord $A C$ ).

Proof OF (i). Let $\widetilde{P}_{1} P_{2}$ be any subarc of (2), where $P_{1}:\left(k \cdot \cos \alpha_{1}, \alpha_{1}\right)$, $P_{2}:\left(k \cdot \cos \alpha_{2}, \alpha_{2}\right),-\pi / 2<\alpha_{1}<\alpha_{2} \leqq \pi / 2$. If $\alpha_{1}<0<\alpha_{2}$, then the point $(k, 0)$ satisfies (i). In each of the remaining cases, $-\pi / 2<\alpha_{1}<\alpha_{2} \leqq 0$ and $0<\alpha_{1}<\alpha_{2} \leqq \pi / 2$, which are now considered, it is noted that $\cos \alpha_{1} \neq \cos \alpha_{2}$. Let the (unordered) set $\left\{\cos \alpha_{1}, \cos \alpha_{2}\right\}$ be denoted by $\{\lambda, \mu\}$ where $0 \leqq \lambda<\mu \leqq 1$. A rational number $W$ can be selected such that

$$
\left(\frac{1-\mu}{1+\mu}\right)^{1 / 2}<W<\left(\frac{1-\lambda}{1+\lambda}\right)^{1 / 2}
$$

Then

Received by the editors October 16, 1961. 


$$
\lambda<\frac{1-W^{2}}{1+W^{2}}<\mu
$$

Let

$$
\phi= \begin{cases}-\operatorname{Arccos} \frac{1-W^{2}}{1+W^{2}} & \text { if } \alpha_{1}<\alpha_{2} \leqq 0, \\ +\operatorname{Arccos} \frac{1-W^{2}}{1+W^{2}} & \text { if } 0<\alpha_{1}<\alpha_{2} .\end{cases}
$$

Then $\phi \in R$, and thus the point $Q:(k \cdot \cos \phi, \phi) \in S$. Since $\cos \phi$ lies between $\cos \alpha_{1}$ and $\cos \alpha_{2}$ (cf. (3)) and the function $\cos x$ is monotonic on $-\pi / 2<x \leqq 0$ and on $0<x \leqq \pi / 2$, the point $Q$ is on $\widetilde{P_{1} P_{2}}$.

Proof of (ii). Let $O$ denote the pole, and $P_{1}:\left(k \cdot \cos \theta_{1}, \theta_{1}\right)$, $P_{2}:\left(k \cdot \cos \theta_{2}, \theta_{2}\right)\left(\theta_{1}<\theta_{2}\right)$ be any two points in $S$. Since angle $P_{1} O P_{2}$ equals $\left(\theta_{2}-\theta_{1}\right),\left|P_{1} P_{2}\right|=k \cdot \sin \left(\theta_{2}-\theta_{1}\right)$. Thus the distance between any two points in $S$ is rational. Consequently, any triangle having vertices in $S$ has rational sides, and, since $k$ is rational, rational area (cf. (1)). Therefore (ii) holds.

Proof of (iii). Let $A C P Q$ be the rectangle circumscribed about $\overparen{A C A}$ (i.e. $P Q$ is tangent to $\overparen{A C}$ at its midpoint). Let $M$ and $N$ be the midpoints of $A Q$ and $C P$, respectively, and denote the points of intersection of $M N$ with $\overparen{A C}$ by $A^{\prime}$ and $C^{\prime}$. A point $B \in S$ can be selected on the minor arc. $\overparen{A^{\prime} C^{\prime}}$ (cf. (i)). The area of $\triangle A B C$ exceeds that of $\triangle A M C$, namely, one-fourth the area of the rectangle $A C P Q$. Since the area of the rectangle $A C P Q$ exceeds that of the segment about which it is circumscribed, (iii) holds.

Let $T$ be the set of all triangles having elements of $S$ as vertices; every element of $T$ is a rational triangle (cf. (ii)). It will now be established that (2) can be rationally triangulated by a subset of $T$.

In view of (i) and (ii) an acute triangle $T_{0} \in T$ can be selected. In each minor segment of (2) subtended by a side $X Y$ of $T_{0}$, a point

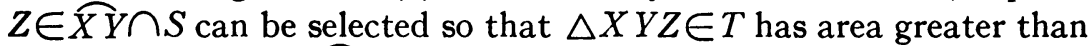
one-fourth that of $\widehat{X Y X}$ (cf. (iii)); thus triangles $T_{i} \in T(i=1,2,3)$ are obtained. Operating similarly on each segment of (2) subtended by a side of the boundary of $T_{0} \cup T_{1} \cup T_{2} \cup T_{3}$ (considering $T_{i}$ as closed regions), triangles $T_{i} \in T(i=4,5, \cdots, 9)$ are obtained; operating similarly on $T_{0} \cup \cdots \cup T_{9}$, triangles $T_{i} \in T(i=10,11, \cdots, 21)$ are obtained; etc.

Thus, if $t_{i}$ denotes the area of $T_{i}(i=0,1,2, \cdots)$, and $u_{j}=3\left(2^{j}-1\right)$, then

$$
\frac{\pi k^{2}}{4}-\sum_{i=0}^{u_{n}} t_{i}<\frac{3}{4}\left(\frac{\pi k^{2}}{4}-\sum_{i=0}^{u_{n-1}} t_{i}\right) \quad(n=1,2,3, \cdots) .
$$


Consequently,

$$
\frac{\pi k^{2}}{4}-\sum_{i=0}^{u_{n}} t_{i}<\left(\frac{3}{4}\right)^{n}\left(\frac{\pi k^{2}}{4}-t_{0}\right)
$$

and hence,

$$
\lim _{m \rightarrow \infty} \sum_{i=0}^{m} t_{i}=\frac{\pi k^{2}}{4} .
$$

Thus, the set $\left\{T_{0}, T_{1}, T_{2}, \cdots\right\}$ is a rational triangulation of (2). This completes the proof of the theorem.

REMARKs. (1) The triangulation $\left\{T_{0}, T_{1}, T_{2}, \cdots\right\}$ is locally finite in the sense that any circle inside (2) intersects only a finite number of the $T_{i}$. It is possible to construct rational triangulations of (2) which are not locally finite.

(2) A "rational refinement" may be obtained by joining the midpoints of the sides of each $T_{i}$. Another rational refinement (and extension) technique is given in [1].

As an immediate consequence of the theorem, a result is obtained apropros the following generally unresolved problem: If $C_{1}, C_{2}, \cdots$, $C_{n}$ are $n$ circles in a plane, is it possible to find points $P_{1}, P_{2}, \cdots, P_{n}$ inside $C_{1}, C_{2}, \cdots, C_{n}$, respectively, such that the distance between $P_{i}$ and $P_{j}$ (for all $i, j$ ) is rational? (L. J. Mordell has answered this question in the affirmative for $n=4$ (cf. [2]).)

CoROllaRy. If a circle $K$ passes through interior points of each of the $n$ circles $C_{1}, \cdots, C_{n}$, then it is possible to find points $P_{1}, \cdots, P_{n}$ inside $C_{1}, \cdots, C_{n}$ respectively, such that the distance between $P_{i}$ and $P_{j}($ for all $i, j)$ is rational.

Proof. Since $K$ passes through interior points of each $C_{i}$, then it is possible to find a circle $K^{\prime}$ with rational radius which passes through interior points of each $C_{i}$. The corollary is an immediate consequence of (i) and (ii).

\section{REFERENCES}

1. F. Supnick, Rational triangulations, Amer. Math. Monthly 68 (1961), 95-102.

2. L. J. Mordell, Rational quadrilaterals, J. London Math. Soc. 35 (1960), 277-282.

The City College 